Original Article

\title{
Brucellosis: Evaluation of Two Hundred and Ten Cases with Different Clinical Features
}

Esma Eroglu, ${ }^{1} M D$, Bahar Kandemir, ${ }^{2} M D$

\begin{abstract}
Introduction: Brucellosis is an important zoonotic disease and a major cause of morbidity worldwide presenting with varying clinical manifestations. We aimed to investigate the epidemiological characteristics and complications of brucellosis at the Van Training and Research Hospital from January 2018 to December 2018. Materials and Methods: 210 patients with brucellosis were assessed retrospectively. The clinical histories and signs, laboratory findings, therapeutic features and complications of these patients were obtained by examining their medical files. Results: Of the 210 patients, the most common symptoms were myalgia $(87.6 \%)$, arthralgia $(76.2 \%)$ and fever $(\mathbf{7 2 . 4 \% )}$. The most frequent clinical signs were fever $(\mathbf{7 2 . 4 \% )}$ ) and arthritis $(\mathbf{2 1 . 9 \% )}$. Out of 210 patients, standard tube agglutination (STA) tests werepositive in 208 (99\%). Osteoarticular involvement $(34.3 \%)$ was common; and a combination of rifampicin and doxycycline was the most frequently used antimicrobial regimen. Conclusion: It should be kept in mind that brucellosis may occur in patients presenting with fever, sweating, arthralgia and leukopenia, especially in endemic areas where dairy livestock is prevalent. People living in these areas should be educated about proper animal care and the handling of dairy products.
\end{abstract}

Ann Acad Med Singapore 2020;49:462-67

Key words: Brucella infection, Turkey, Zoonosis

\section{Introduction}

Brucellosis is an important zoonosis in the world. ${ }^{1}$ In Turkey, brucellosis is common, especially in the Mid, East and Southeast Anatolia regions. The Brucella bacteria is transmitted to humans from raw milk and dairy products, raw meat and infected animal products. ${ }^{2,3,4}$ Brucellosis is still a major cause of morbidity in humans and remains a major public health problem in Turkey presenting with a wide range of clinical manifestations. Fever, sweating, fatigue, osteoarthritis can be seen in patients with their hematological, musculoskeletal, urogenital and central nervous systems affected. ${ }^{5}$ It can cause serious complications, namely spondyloarthritis and epididymo-orchitis, the musculoskeletal system involvement being the most frequently seen complication. Its broad spectrum of clinical presentations leads to delay in diagnosis and treatment. ${ }^{6}$ The purpose of our study is to analyse the epidemiological, clinical, and laboratory findings and therapeutic features of our patients with brucellosis and compare our results with other similar studies in the literature.

\footnotetext{
${ }^{1}$ Department of Infectious Diseases and Clinical Microbiology, Konya Training and Research Hospital, Konya, Turkey

${ }^{2}$ Department of Infectious Diseases and Clinical Microbiology, Meram Faculty of Medicine, Necmettin Erbakan University, Konya, Turkey

Address for Correspondence: Dr Esma Eroğlu, Department of Infectious Diseases and Clinical Microbiology, Konya Training and Research Hospital, Yeni Meram Street, No:97, 42090, Konya, Turkey

Email:esmagulesen@hotmail.com
} 


\section{Materials and Methods}

Two hundred and ten patients diagnosed and treated for brucellosis from January 2018 to December 2018 in the Department of Infectious Diseases and Clinical Microbiology were evaluated retrospectively. Both male and female patients above the age of 18 years were included in the study. Six patients were pregnant. Patients' histories, clinical and laboratory findings, therapeutic features and complications were obtained from their medical files. The patients were diagnosed with brucellosis if positive clinical signs and symptoms were accompanied by either isolation of Brucella bacteria in blood culture, or presence of positive titers (above 1/160) in standard tube agglutination test (STA) and 2-fold increase in the STA taken at 2-week intervals. STA (Cromatest, Linear Chemicals, Spain) measuring antibody to Brucella bacteria was performed by dilution in a tube, incubated for 24 hours at $37^{\circ} \mathrm{C}$, and antibody titer was evaluated according to agglutination. A value above 1/160 titres was considered as positive. Automated culture identification system BACTEC FX (Becton-Dickinson, Sparks, MD, USA) was used for blood cultures.After positive signals passage was made from the blood culture bottles, Gram stains and biochemical tests were performed from the colonies grown as a result of incubation for 48 hours. Brucella bacteria were identified as catalase and oxidase positive Gramnegative coccobacilli.

Brucellosis is classified into 3 groups depending on duration of symptoms: acute (0-2 months), subacute (2-12 months) and chronic (>12 months). ${ }^{7}$ Radiologic evaluations, such as X-ray, ultrasound (USG) and magnetic resonance imaging (MRI) were used if required to investigate for complications. These methods were chosen based on presence of symptoms and signs of patients. Osteoarticular involvement was considered when there was pain, warmth, redness, functional disability in peripheral joints, hips or spine. Epididymoorchitis was confirmed by USG if pain and redness were present in patient's scrotum.

Patients were generally treated with 6 weeks of a combination therapy of oral doxycycline $(100 \mathrm{mg}$ every $12 \mathrm{~h})+$ rifampicin (600 mg every 24 hours); doxycycline (100mg every 12 hours) +21 days of streptomycin ( $1 \mathrm{~g}$ every 24 hours) or 7 days gentamicin $(5 \mathrm{mg} / \mathrm{kg}$ every 24 hours), doxycycline (100mg every 12 hours) + trimethoprim-sulfamethoxazole (160/800 every 12 hours), rifampicin (600mg every 24 hours) + trimethoprim-sulfamethoxazole (160/800 every 12 hours), doxycycline (100mg every 12 hours) + ciprofloxacin (500mg every 12 hours), rifampicin (600mg every 24 hours) + ceftriaxone (1g every 12 hours). Aminoglycoside combination was used mostly in osteoarticuler involvement and ceftriaxone combination was used in pregnancy. Patients were evaluated at 2-week intervals with treatment, treatment extended if necessary. Furthermore, after completing antibiotic therapy, all patients were followed up for a year. The first assessment of the patients was performed after 2 weeks followed by 6 weeks, 12 weeks, 6 months and 1 year later. STA was conducted again after antibiotic therapy and then repeated if the symptoms reappeared. Complete blood count, C-reactive protein (CRP), erythrocyte sedimentation rate (ESR), and liver enzymes were analysed and repeated during follow-up of the patients. After treatment, the reappearance of symptoms or 2-fold increase in the STA values taken at 2-week intervals was documented as a relapse. However, relapses presented to other hospitals were excluded.

SPSS version 20 was used for statistical analysis. The Student's t-test and Fisher's exact test were used for categorical analysis, with $P<0.05$ considered to be statistically significant.

\section{Results}

Of the 210 patients, $128(61 \%)$ were female and $82(39 \%)$ were male. The mean age of patients was 38 years (range 18-85). 76 of patients $(36.2 \%)$ had a history of animal husbandry, and 182 of patients $(86.7 \%)$ had a history of consumption of raw milk and dairy products. One of the patients had laboratoryrelated contact $(0.48 \%)$, and 12 of the patients $(5.7 \%)$ were found to have no known risk factor for brucellosis. The common clinical symptoms were myalgia (87.6\%), arthralgia $(76.2 \%)$ and fever $(72.4 \%)$. The average time from the onset of symptoms to the hospital admission was 4 days. The time to improvement in 152 patients with fever was on average 3 days. The common clinical signs were fever $(72.4 \%)$, arthritis $(21.9 \%)$ and hepatomegaly $(20 \%)$. Osteoarticular involvement was present in 72 patients $(34.3 \%)$, including arthritis $(21.9 \%)$, spondylitis (5.7\%) and sacroiliitis (6.7\%). Endovascular infections and neurobrucellosis were not detected during this period. Brucella bacterial growth in blood culture was achieved in 22 of 70 cases. Typing was possible for only 18 isolates; 14 of them were brucella melitensis and 4 were brucella abortus. The clinical and laboratory features of the patients are presented in Tables 1 and 2. 
Table 1. The Clinical Features (Symptoms and Signs) of Patients

\begin{tabular}{lcc}
\hline Symptoms & n (210) & \% \\
\hline Fever & 152 & 72.4 \\
\hline Sweating & 124 & 59 \\
\hline Arthralgia & 160 & 76.2 \\
\hline Myalgia & 184 & 87.6 \\
\hline Fatigue & 124 & 59 \\
\hline Back pain & 78 & 37.1 \\
\hline Hip pain/walking difficulty & 31 & 14.8 \\
\hline Weight loss & 32 & 15.2 \\
\hline Scrotal pain & 13 & 6.2 \\
\hline Signs & 14 & 6.7 \\
\hline Hepatomegaly & 13 & 20 \\
\hline Splenomegaly & 42 & 16.2 \\
\hline Arthritis & 34 & 5.7 \\
\hline Spondylitis & 46 & \\
\hline Sacroiliitis & 12 & 6.9 \\
\hline Epididymo-orchitis & & \\
\hline
\end{tabular}

Table 2. Laboratory Features of the Patients

\begin{tabular}{lcc}
\hline & n (210) & \% \\
\hline Anemia $(\mathrm{Hb}<10 \mathrm{~g} / \mathrm{dl})$ & 68 & 32.4 \\
Leukocytosis $(>10000 / \mathrm{mm} 3)$ & 32 & 15.2 \\
Leokopenia $(<4000 / \mathrm{mm} 3)$ & 78 & 37.1 \\
\hline Thrombocytopenia $(<150000 / \mathrm{mm} 3)$ & 38 & 18.1 \\
ALT elevation $(>40 \mathrm{iu} / \mathrm{l})$ & 78 & 37.1 \\
CRP elevation $(>8 \mathrm{~g} / \mathrm{dl})$ & 122 & 58.1 \\
\hline ESR elevation $(>20 \mathrm{~mm} / \mathrm{h})$ & 138 & 65.7 \\
STA positive & 208 & 99 \\
\hline Cultures positive/cultures taken & $22 / 70$ & $31.4 *$ \\
\hline
\end{tabular}

Alanine aminotransferase (ALT), C-reactive protein (CRP), erythrocyte sedimentation rate (ESR), standard tube agglutination (STA)

*culture positivity rate of total cultures taken

The treatment regimens were a 6-week combination of oral doxycycline+rifampicin, 6 weeks of doxycycline +21 days of streptomycin or 7 days gentamicin, 6 weeks of doxycycline+trimethoprim-sulfamethoxazole, 6 weeks of rifampicin+trimethoprim-sulfamethoxazole,
6 weeks of doxycycline + ciprofloxacin, 6 weeks of rifampicin+ ceftriaxone. Aminoglycoside combination was used mostly in osteoarticulear involvement and ceftriaxone combination was used in pregnancy. In pregnant women, intravenous ceftriaxone ( $2 \mathrm{~g}$ per day) was added to the regimen initially for $2-4$ weeks, and other antimicrobials were given for at least 6 weeks. When required, the duration of therapy was extended and data were recorded. The medication period was 6-12 weeks in osteoarticular involvement. In chronic brucellosis, the medication period was extended to 12-24 weeks. The patients were treated on an average of 88 (range 45-380) days. After completing antibiotic therapy,only 138 of 210 patients were followed up to 1 year. Relapse was detected in 8 patients $(3.8 \%) ; 6$ of them $(2.9 \%)$ were patients with osteoarticular involvement treated with a regimen of aminoglycoside combination, 2 of them $(0.9 \%)$ had no complications. The medications used and the relapse rate of the patients are evaluated in Table 3.

Of the 210 patients, $72 \%$ of the cases were acute, $24 \%$ were subacute and $4 \%$ were in chronic form. Their clinical symptoms and signs are presented in Table 4.

\section{Discussion}

In Turkey, brucellosis is still an endemic disease and an important public health problem. Limited veterinary support services and husbandry practices favored the spread of infection. Moreover, in recent years, there has also been uncontrolled immigration from surrounding countries such as Syria, Iran, Iraq where brucellosis is endemic. ${ }^{8}$ Both genders are equally affected $^{9,10}$ though brucellosis has long been recognised as predominantly affecting adult men. However, in our study, more women were affected. Some

Table 3. Medications Used and Relapse Rate of the Patients

\begin{tabular}{lcc}
\hline Medications & n (\%) & Relapse (\%) \\
\hline Doxycycline + rifampicin & $108(51.4)$ & $2(0.9)$ \\
$\begin{array}{l}\text { Doxycycline + streptomycin/ } \\
\text { gentamicin }\end{array}$ & $72(34.3)$ & $6(2.9)$ \\
$\begin{array}{l}\text { Doxycycline + trimethoprim- } \\
\text { sulfamethoxazole }\end{array}$ & $24(11.4)$ & 0 \\
$\begin{array}{l}\text { Doxycycline + ciprofloxacin } \\
\text { Doxycycline + ceftriaxone }\end{array}$ & $4(1.9)$ & 0 \\
\hline Total & $210(100)$ & $8(3.8)$ \\
\hline
\end{tabular}


Table 4. Comparison of Brucellosis Phases

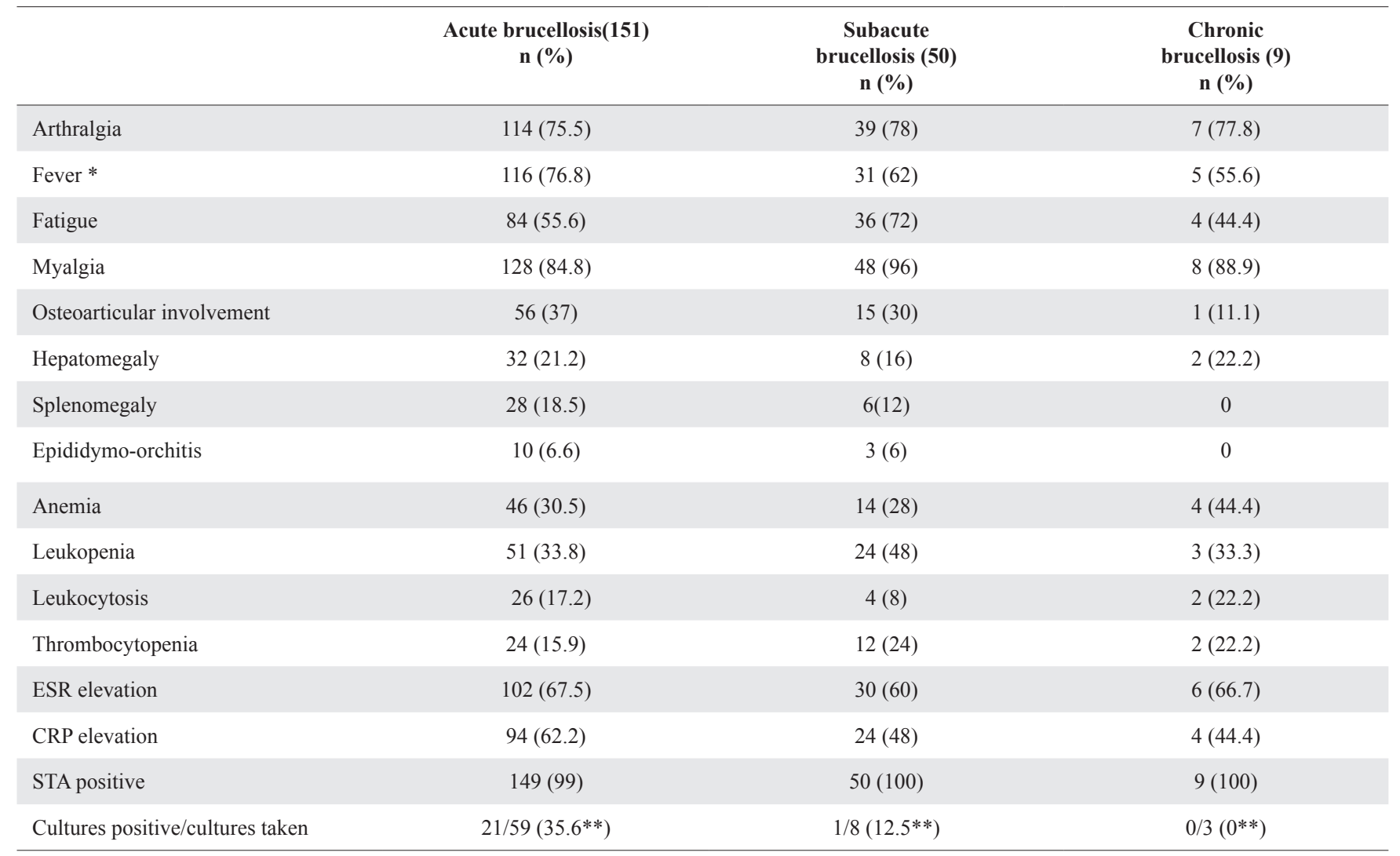

$* P=0.001$

$* * \%$ culture positivity of the culture taken patients

investigators have reported more severe forms of disease in women but these findings have yet to be affirmed..$^{10,11}$

It has been shown that $63.6 \%$ of Brucellosis patients in Turkey had consumed raw animal products. ${ }^{5}$ We determined that $86.7 \%$ of our patients were infected through the consumption of raw animal products. The laboratory staff working with these bacteria is also at risk of contamination. However, laboratory-acquired brucellosis cases are rarely reported in our country. ${ }^{12}$ In our study, only one $(0.48 \%)$ patient was related to laboratory contact. Brucellosis can present with nonspecific clinical manifestations: fever, fatigue, myalgia, hepatomegaly or splenomegaly. Fever was the most common clinical finding in our study as per literature. Among the complications of brucellosis, osteoarticular involvement is the most common complication. However, its prevalance has been reported to vary widely from $1 \%$ to $69 \% .^{11}$ In our study, arthritis is the mostcommon finding. The diversity of criteria used for the detection of skeletal involvement in brucellosis may have led to the wide variations in the rates reported by different researchers.

Brucellosis is classified into 3 groups depending on duration of symptoms: acute, subacute and chronic. ${ }^{7}$ Of the 210 patients, $72 \%$ wereacute, $24 \%$ subacute and $4 \%$ chronic. Several studies have found acute brucellosis in $25-77 \%$, subacute brucellosis in $12.5-59 \%$ and chronic brucellosis in $5-27.5 \%$ of their patients. ${ }^{13,14}$ Brucellosis may present with a wide variety of clinical signs and symptoms. Symptoms in acute brucellosis are more pronounced and severe with fatigue, sweating, arthralgia and myalgia being common. Some untreated patients with acute brucellosis may progress into subacute phase with symptoms extending from 2 months to a year. Subacute cases demonstrated less severe symptoms when compared with the acute form. Chronic brucellosis is defined as the persistence of symptoms related to infection for more than 1 year. Physical 
findings in these patients are not as high as in the acute or subacute cases with fatigue, insomnia, emotional lability, myalgia being prominent. Such differences are associated with the cultural and socio-economic status of the patient, and also associated with the differences in the way the patients were diagnosed. Since the acute form is more common, early suspicion of symptoms is therefore important in early diagnosis.

The results of our study did not show any significant difference between the form of the disease in regards with arthritis, arthralgia, splenomegaly, and hepatomegaly. Fever was however significantly $(P=0.001)$ more frequent in acute brucellosis compared to the other disease forms (Table 4). When the relationship between the disease forms was assessed, there was no significant association with osteoarticuler involvement. Spondylitis (rare in the acute form) was detected more in the subacute and chronic forms, whereas epididymo-orchitis (present in the acute and subacute forms) was absent in the chronic form. Other studies in Turkey had shown that fever was observed in $76.9 \%$ of the acute cases, in $65.7 \%$ of the subacute cases and in $57.6 \%$ of chronic brucellosis. It had also been reported that hepatomegaly and splenomegaly were less common in chronic brucellosis compared to acute and subacute brucellosis, however spondylitis was more frequent in the chronic form. ${ }^{10}$ In our study, there was no splenomegaly in the chronic brucellosis cases.

Osteoarticular involvement (such as arthritis, spondylitis and sacroiliitis) is the most frequent complication with a frequency of $21 \%$ to $37.5 \% .^{5,15,16}$ Similar to previous studies, osteoarticular manifestations were observed $34.3 \%$ of our patients. The difference in prevalence between studies may be related to the undetected complications, but there was no statistically significant difference in relationship between the disease forms.

Similiar to other studies, leukopenia, anemia and thrombocytopenia were also common in our study. ${ }^{17}$ Furthermore, elevated liver enzymes and CRP were mostly seen in acute and subacute brucellosis.An increase in ESR was more frequent in chronic brucellosis; however, ESR did not demonstrate any significant difference between the different forms of the disease in our study. The initial method for the detection of brucellosis is serology whereas positive culture is used to confirm the diagnosis. In Turkey, the serologic test is commonly used in the diagnosis of brucellosis. In our study, serology positivity rate was $99 \%$ of patients. Only 2 patients had negative STA tests at first, but their infection was later confirmed with positive blood cultures. The rate of positive blood cultures in brucellosis varied from $15-80 \% .^{18}$ Acute brucellosis usually has a higher frequency of positive culture while negative results are particularly seen with the chronic form of the disease. Similarly, in our study, acute brucellosis had a higher frequency of positive culture but none in chronic form of the disease. ${ }^{19}$ Various drug combinations were used to treat our patients with brucellosis. As per standard treatment, the most commonly used treatment regimen was doxycycline and rifampicin (51.4\%) as recommended by the World Health Organization. We chose treatments based on clinical presentations of the disease. In osteoarticulear involvement, doxycycline + streptomycin combination is recommended and more effective than the doxycycline + rifampicin combination. ${ }^{20}$ In our study, the doxycycline + streptomycin combination was shown to be more effective than other combinations in osteoarticular involvement. In 1 study,the relapse rate was $4.7 \%$ and the highest rate was seen in patients with osteoarticular involvement. ${ }^{5}$ Similarly, our relapse rate was $3.8 \%$ and was most frequently detected in patients with osteoarticular involvement. The limitation of our study was the retrospective documentation of relapse rate based on medical notes and 72 patients were lost to follow-up.

\section{Conclusion}

In conclusion, brucellosis is still an important zoonosis in Turkey. It should be kept in mind that patients may present with non-specific fever, sweating, arthralgia and leukopenia, especially in the endemic areas where dairy livestock is common. People living in these areas should be educated regarding the risk of brucellosis during animal care and dairy product consumption. Control of the disease in animals is also important for the control of human brucellosis. A multidisciplinary approach involving veterinary, agricultural besides healthcare services is necessary for the proper treatment in brucellosis.

\section{REFERENCES}

1. Zheng R, Xie S, Lu X, Sun L, Zhou Y, Zhang Y et al. A Systematic Review and Meta-Analysis of Epidemiology and Clinical Manifestations of Human Brucellosis in China. BioMed Res Int. 2018:22.

2. De MF, Di GA, Petrini A, Pizzigallo E, Giovannini A. Correlation between animal and human brucellosis in Italy during the period 1997-2002. Clin Microbiol Infect. 2005;11:632-6.

3. Mangalgi S, Sajjan AG, Mohite ST, Gajul S. Brucellosis in occupationally exposed groups. JClin Diagn Res. 2016;10:DC24-7. 
4. Moosazadeh M, Nikaeen R, Abedi G, Kheradmand M, Safiri S. Epidemiological and clinical features of people with Malta fever in Iran: a systematic review and meta-analysis. Osong Public Health Res Persp.2016;7:157-67.

5. Buzgan T, Karahocagil MK, Irmak H, Baran AI, Karsen H, Evirgen $\mathrm{O}$,et al. Clinical manifestations and complications in 1028 cases of brucellosis: a retrospective evaluation and review of the literature. Int J Infect Dis. 2010;14:469-78.

6. Jiang W, Chen J, Li Q, Jiang L, Huang Y, Lan Y et al. Epidemiological characteristics, clinical manifestations and laboratory findings in 850 patients with brucellosis in Heilongjiang Province, China. BMC Infect Dis. 2019;19:439.

7. Guland HC, Erdem H. Brucellosis (Brucella Species). Mandell, Douglas, And Bennett's Principles and Practice of Infectious Diseases. 9th Ed. Philadelphia (PA): Elsevier. 2020: 2753-58.

8. Kozat S, Ozbek M. Brucellosis. Turk J Vet Anim Sci. 2019;1:10-8.

9. Ariza J, Gudiol F, Valverde J,Pallares P, Fernandez-Viladrich P, Rufi G, et al. Brucellar spondylitis: detailed analysis based on current findings. Rev Infect Dis.1985;7:656-64.

10. Turgut H, Hoşoğlu S, Aydın K, Arıturk S. Clinical and laboratory findings in 98 patients. Med J Ege Univ. 1991;1(1):153-57.

11. Rajapakse CN. Bacterial Infections: Osteoarticular Brucellosis. Bailieres Clin Rheumatol. 1995;9:161-77.

12. Demirdal T, Demirtürk N. Laboratory-acquired brucellosis. Ann Acad Med Singapore. 2008;37:86-7.
13. Gur A, Geyik MF, Dikici B, Nas K, Cevik R, Sarac J, et al. Complications of brucellosis in different age groups: a study of 283 cases in Southeastern Anatolia of Turkey. Yonsei Med J. 2003;44:33-44.

14. Mantur BG, Biradar MS, Bidri RC, Mulimani MS, Veerappa K, Kariholu $\mathrm{P}$,et al. Protean clinical manifestations and diagnostic challenges of human brucellosis in adults: 16 years' experience in an endemic area. J Med Microbiol. 2006;55:897-903.

15. Agalar C, Usubutun S, Turkyilmaz R. Ciprofloxacin and rifampicin versus doxycycline and rifampicin in the treatment of brucellosis. Eur J Clin Microbiol Infect Dis. 1999;18:535-8.

16. Colmenero JD, Reguera JM, Martos F, et al. Complications associated with Brucella melitensis infection: a study of 530 cases. Medicine (Baltimore). 1996;75:195-211.

17. Doganay M, Aygen B. Human brucellosis: an overview. Int J Infect Dis. 2003;7:173-82.

18. M.J. Corbel. Brucellosis: an overview. Emerg Infect Dis. 1997;3:213-21.

19. Solera J, Rodriguez-Zapata M, Geijo P, Largo J, Paulino J, Saez L,et al. Doxycycline-rifampin versus doxycycline-streptomycin in treatment of human brucellosis due to Brucella melitensis. Antimicrob Agents Chemother. 1995;39:2061-67.

20. Ariza J, Gudiol F, Pallares R, Viladrich PF, Rufi G, Corredoira J et al. Treatment of human brucellosis with doxycycline plus rifampin or doxycycline plus streptomycin. A randomized, double-blind study. Ann Intern Med. 1992;117:25-30. 